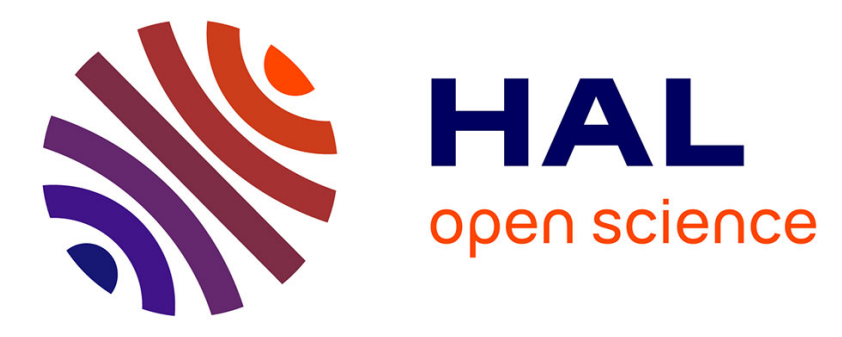

\title{
Magnus-force model for active particles trapped on superfluid vortices
}

Adam Griffin, Vishwanath Shukla, Marc-Etienne Brachet, Sergey Nazarenko

\section{To cite this version:}

Adam Griffin, Vishwanath Shukla, Marc-Etienne Brachet, Sergey Nazarenko. Magnus-force model for active particles trapped on superfluid vortices. Physical Review A, 2020, 101 (5), pp.053601. 10.1103/PhysRevA.101.053601 . hal-03079540

\section{HAL Id: hal-03079540 https://hal.science/hal-03079540}

Submitted on 17 Dec 2020

HAL is a multi-disciplinary open access archive for the deposit and dissemination of scientific research documents, whether they are published or not. The documents may come from teaching and research institutions in France or abroad, or from public or private research centers.
L'archive ouverte pluridisciplinaire HAL, est destinée au dépôt et à la diffusion de documents scientifiques de niveau recherche, publiés ou non, émanant des établissements d'enseignement et de recherche français ou étrangers, des laboratoires publics ou privés. 


\title{
Magnus-force model for active particles trapped on superfluid vortices
}

\author{
Adam Griffin, ${ }^{1,5,{ }^{*}}$ Vishwanath Shukla,,${ }^{2,3, \dagger}$ Marc-Etienne Brachet $\odot,{ }^{4}$ and Sergey Nazarenko \\ ${ }^{1}$ Mathematics Institute, The University of Warwick, Coventry, CV4 7AL, United Kingdom \\ ${ }^{2}$ Department of Physics, Indian Institute of Technology Kharagpur, Kharagpur 721 302, India \\ ${ }^{3}$ Centre for Theoretical Studies, Indian Institute of Technology Kharagpur, Kharagpur 721302, India \\ ${ }^{4}$ Laboratoire de Physique de l'Ecole Normale Supérieure, ENS, Université PSL, CNRS, Sorbonne Université, Université de Paris, F-75005 \\ Paris, France \\ ${ }^{5}$ Institut de Physique de Nice, Université Côte d'Azur, CNRS, Nice 06108, France
}

(Received 22 September 2019; revised manuscript received 27 March 2020; accepted 1 April 2020;

published 1 May 2020)

\begin{abstract}
Experimentalists use particles as tracers in liquid helium. The intrusive effects of particles on the dynamics of vortices remain poorly understood. We implement a study of how basic, well understood vortex states, such as a propagating pair of oppositely signed vortices, change in the presence of particles by using a simple model based on the Magnus force. We focus on the two-dimensional case, and compare the analytic and semianalytic models with simulations of the Gross-Pitaevskii (GP) equation with particles modeled by dynamic external potentials. The results confirm that the Magnus force model is an effective way to approximate vortex-particle motion either with closed-form simplified solutions or with a more accurate numerically solvable ordinary differential equations. Furthermore, we increase the complexity of the vortex states and show that the suggested semianalytical model remains robust in capturing the dynamics observed in the GP simulations.
\end{abstract}

DOI: 10.1103/PhysRevA.101.053601

\section{INTRODUCTION}

Superfluidity occurs in a wide variety of systems, including terrestrial ones-e.g., ${ }^{4} \mathrm{He}$ II [1], ${ }^{3} \mathrm{He} \mathrm{B}$ [2], versatile Bose-Einstein condensates (BECs) of alkali atoms [3], and exciton-polariton condensates [4] in laboratory experimentsand exotic astrophysical objects, such as neutron stars [5,6]. Moreover, a monochromatic light while passing through a nonlinear medium, e.g., photorefractive crystals [7], has been shown to exhibit a flow that is essentially superfluid. The unusual flow properties of these superfluid flows have held the attention of experimentalists and theorists alike. For example, helium II can sustain rotational motion only through formation of quantized vortices, wherein the circulation along paths enclosing vortices is restricted to multiples of $h / m_{4}$, where $h$ is Planck's constant and $m_{4}$ is the mass of a ${ }^{4} \mathrm{He}$ atom [8]. These quantized vortices in helium II are angstrom size in diameter, and they occur either as closed loops or filaments that must end at the boundary of the fluid.

Quantized vortices display rich dynamical behavior [9]; for example, in three-dimensional (3D) rotating superfluid systems above the vortex nucleation threshold where the number of vortices increases with rotation speed and can form a vortex lattice. At still higher rotation speed the system transitions to a turbulent state [10]. It is a nonequilibrium state involving processes that span a broad range of length and time scales, and is characterized by the presence of a dynamic random tangle of interacting vortices [11-13]. These

\footnotetext{
*a.griffin.1@warwick.ac.uk

${ }^{\dagger}$ research.vishwanath@gmail.com
}

quantized vortices upon close approach can undergo reconnections $[14,15]$, a topology changing process that further drives the system to a turbulent state. Reconnection events excite Kelvin waves on the vortices, and the nonlinear interaction of these waves gives rise to a cascade process that transfers energy to smaller length scales, which is ultimately radiated as sound [16-19]. Turbulence in these superfluid systems can also be excited by stirring, shaking, moving objects, etc. [1,3,20]. Moreover, turbulent states can also be realised in two-dimensional (2D) superfluids [21,22], where pointlike vortices move chaotically [23-25] and can organize to produce large-scale flows. This subject has seen a spurt of recent activity, both numerical and experimental, to find its universal features and provide a comparison with its classical counterpart [26-28].

However, it must be emphasised that the experimental study of the fundamental processes involved in superfluid turbulence is a difficult task and requires state-of-the-art facilities $[8,29]$. In particular, visualization of the quantized vortices has been a challenge because of extremely low temperatures and small system sizes. The flow visualization methods available for classical fluids are difficult to adapt to superfluid helium [30].

Use of particles to probe superfluid flows involving vortices was suggested in Ref. [31]. A great deal of information about quantized vortices, including their existence, in helium II has been obtained, in the past, by the use of moving ions [32]. More recently, solid-hydrogen particles were used to visualize quantized vortices in helium II at temperatures $\sim 2 \mathrm{~K}[33,34]$. These particles were also used to study vortex reconnection events [14] and Kelvin waves [18] on vortices in 
superfluid helium. Note that in these experiments, even though particles used were roughly $10^{4}$ times the vortex diameter, they managed to capture the essential physics.

Now much smaller particles in the form of metastable ${ }^{4} \mathrm{He}_{2}^{*}$ excimer molecules, $\sim 100 \mathrm{~nm}$ in size, are available that can be used as vorticity tracers in the $T=0$ limit, in the absence of normal fluid; at temperatures above $1 \mathrm{~K}$ they act as tracers of the normal component [35].

Notwithstanding the significant experimental progress in the use of particles to characterize superfluid turbulence, the exact level of intrusion of the particle on the vortex motion remains unclear. Therefore, it is important to explore and understand these issues by building simplified models to study particle-vortex dynamics both theoretically and numerically [36,37].

The models presented may also be relevant to BECs with impurities [38] and optical fluid of light experiments [7] with multiple beams interacting.

In this work, we focus on the Magnus force that acts on a particle trapped on a translating vortex. To do so, we make use of both the Gross-Pitaevskii (GP) equation description of superfluids $[39,40]$ and the suitably adapted classical treatment of point vortices in 2D. In our GP description, we make use of a recently developed minimal model [41-43], wherein we couple the equations of motion of particles with the GP classical wave function $\psi$. We demonstrate that the Magnus force description borrowed from the theory of ideal hydrodynamic flow provides a good description of the dynamics of vortices loaded with particles in superfluids. We first study a particle loaded vortex-antivortex pair configuration and carry out a systematic direct numerical simulations (DNS) of the GP based minimal model. We compare these GP equation DNS results with a Magnus force model that we have derived (see below), both in the simplified analytically tractable case of constant background flow and a more realistic situation wherein the background flow is allowed to vary in response the particle loaded vortices (or other external vortices). We then extend our study of the particle loaded vortex-antivortex pair to more complex vortex configurations, where (i) each vortex is multiply charged and (ii) free external vortices are present in the neighborhood. We will argue that under certain circumstances the effects can be observed in superfluid helium experiments. Moreover, our study indicates that particles can be a useful tool for future studies of vortex motion in BoseEinstein condensates (BECs).

The advantage of our analytical and semianalytical models is that they can be applied, and work better, for setups with particles much larger than the healing length, whereas it would be virtually impossible to simulate such systems with huge scale separations in DNS. Such models will be useful, for example, in investigations of the effect of hydrogen ice tracers whose typical diameter is the order of a few micrometers [33], while the vortex core diameter is of the order of an angstrom $\left(1 \AA=10^{-10} \mathrm{~m}\right)$. In this situation, we can think of a classical flow with a thin boundary layer, so that one can use classical textbook solutions relating the force on a moving object with the circulation around the object [44]. This indicates that the Magnus model will work better in describing the motion of large particles relative to vortex core size. However, the validity of such a model in quantum fluids cannot be taken for granted due to the presence of the compressibility, acceleration, as well as a flow nonuniformity over the distances comparable to the particle sizes.

In our paper, we demonstrate that the Magnus force model works very well even for situations well beyond the formal limits of applicability of the ideal flow descriptions, e.g., when the size of the particle is no so much bigger than the healing length and the velocity is not so much smaller than the speed of sound.

\section{MODEL AND NUMERICAL METHODS}

\section{A. Gross-Pitaevskii equation coupled with particles}

We use the GP theory to model the superfluid flow and study its interaction with particles. The GP framework provides a good hydrodynamical description of a weakly interacting superfluid at lowtemperatures and is able to reproduce the qualitative features of the strongly interacting superfluid helium. Within this framework, the state of the system is specified by the complex scalar field $\psi(\mathbf{r}, t)$.

The particles that we consider are active; they are affected by flow and act back on it too. In our earlier works [41-43] we introduced a Lagrangian for this combined system, wherein particles were represented by specifying the potential $V_{\mathcal{P}}$. This procedure yields the following GP equation for the spatiotemporal evolution of $\psi(\mathbf{r})$ :

$$
i \hbar \frac{\partial \psi}{\partial t}=-\frac{\hbar^{2}}{2 m_{b}} \nabla^{2} \psi-\mu \psi+g|\psi|^{2} \psi+\sum_{j=1}^{\mathcal{N}_{0}} V_{\mathcal{P}}\left(\mathbf{r}-\mathbf{q}_{j}\right) \psi
$$

where $\hbar=h / 2 \pi$ is the reduced Planck's constant, $m_{b}$ the mass of bosons constituting the superfluid, $g$ the effective interaction strength among these bosons, $\mu$ the chemical potential and $\mathbf{q}_{j}$ the position of the $j$ th particle (i.e., center of the potential).

The strong interactions within liquid helium are not captured by the GP equation. However, phenomenologically the GP equation captures some well known behaviors of liquid helium such as superfluidity and quantized vortices especially in the zero-temperature limit. One main aspect of helium the GP equation does not capture is the dispersion relation and the roton curve. It would be an interesting study to see if modification to the GP equation which have the roton (such as with a nonlocal nonlinearity) affect the predictions of the model.

Our modeling of the particles by specifying $V_{\mathcal{P}}$ allows us to control their characteristics, e.g., shape and size. For the purpose of present study, we use the Gaussian potential

$$
V_{\mathcal{P}}=V_{0} \exp \left(-\frac{r^{2}}{2 d_{p}^{2}}\right)
$$

here $V_{0}$ is the strength of the potential and $d_{\mathrm{p}}$ is the measure of its width. In the presence of another field (noncondensed atoms, another species of BEC, or an obstacle caused by laser or normal fluid, to give some examples) which we can denote the density of fluid trapped in the vortex core, $\rho_{v}$, then $V_{\mathcal{P}} \propto \rho_{v}$. 
Furthermore, we include a two-particle, short-range repulsion potential. Thus, the Newtonian dynamics of the particles is governed by the following equation:

$$
m_{j} \ddot{\mathbf{q}}_{j}=\mathbf{f}_{0, j}+\mathbf{G}_{j},
$$

where $m_{j}$ is the mass of the $j$ th particle, which we assume to be same for all, $m_{j}=m_{0}$, vector $\mathbf{f}_{0, j}$ is the force exerted by the superfluid onto the particle,

$$
\mathbf{f}_{j}=\int_{\mathcal{B}}|\psi|^{2} \nabla V_{\mathcal{P}}\left(\mathbf{r}-\mathbf{q}_{j}\right) d \mathbf{r}
$$

$\mathcal{B}$ is the area occupied by the particle [determined by a cutoff of the potential (2)], and $\mathbf{G}_{j}=\left(G_{x}, G_{y}\right)=$ $\hat{\mathbf{e}}_{x} \sum_{i=1, i \neq j}^{\mathcal{N}_{0}} \frac{\Delta_{E} r_{S R}^{12}}{\left|\left(\mathbf{q}_{i}-\mathbf{q}_{j}\right) \cdot \hat{\mathbf{e}}_{x}\right|^{12}}+\hat{\mathbf{e}}_{y} \sum_{i=1, i \neq j}^{\mathcal{N}_{0}} \frac{\Delta_{E} r_{S R}^{12}}{\left|\left(\mathbf{q}_{i}-\mathbf{q}_{j}\right) \cdot \hat{\mathbf{e}}_{y}\right|^{12}}$ is the interparticle short-range repulsion force. In Ref. [41] it was demonstrated that there is a superfluid mediated attractive force between two particles separated by a small distance (of the order of few of healing lengths); therefore, to model realistic particles we must include a short-range repulsive interaction between particles that for simplicity is inspired by the repulsive part of the widely used Lennard-Jones potential. The parameters $r_{\mathrm{SR}}=5 d_{p}$ and $\Delta_{E}=0.062 c^{2} \xi^{2} \rho_{0}$ together control the strength and the distance of closest approach; we keep these fixed in our study.

\section{B. Numerical methods, units and parameters}

To study the dynamics of particles in complex superfluid flows, we solve Eqs. (1) and (3) numerically. In order to do so, we perform direct numerical simulations (DNSs) of the GP by using the Fourier pseudospectral method on a square, periodic simulation domain $\mathcal{A}$ of side $L$ with $N_{c}^{2}$ collocation points [27]. In this method, we evaluate the linear terms in Fourier space and the nonlinear term in real (physical) space, which we then transform to Fourier space. For the Fouriertransform operations, we use the FFTW library [45]. A fourthorder Runge-Kutta scheme is used to evolve these equations in time. Further details can be found in [43].

We define the length scale $\xi=\hbar / \sqrt{m_{b} g \rho_{0}}$, known as the healing length, and the speed of sound $c=\sqrt{g \rho_{0} / m_{b}}$. The mean density can be also calculated: $\rho_{0}=\int_{\mathcal{A}}|\psi|^{2} d \mathbf{r} / \mathcal{A}$. We then choose to rescale the parameters in (1) with the following scalings: $\tilde{\mu}=\mu /\left(g \rho_{0}\right), \tilde{V}_{\mathcal{P}}=V_{\mathcal{P}} /\left(g \rho_{0}\right), \tilde{\psi}=\psi / \sqrt{\rho_{0}}, \tilde{\mathbf{r}}=$ $\mathbf{r} / \xi$, and $\tilde{t}=t c / \xi$. We arrive at the dimensionless equation which we simulate:

$$
i \frac{\partial \tilde{\psi}}{\partial \tilde{t}}=-\frac{1}{2} \tilde{\nabla}^{2} \tilde{\psi}-\tilde{\mu} \tilde{\psi}+|\tilde{\psi}|^{2} \tilde{\psi}+\sum_{j=1}^{\mathcal{N}_{0}} \tilde{V}_{\mathcal{P}}\left(\mathbf{r}-\mathbf{q}_{j}\right) \psi .
$$

From here onward we drop the tildes for simplicity of notation. Taking into account that we are in dimensionless units, in our calculations we have $\rho_{0}=c=\xi=\mu=1$. In dimensionless units we also choose the parameters $L=177.78$, with the grid spacing $d x=L / N_{c}$, and the number of collocation points $N_{c}=256$. For the external potential we choose the parameters $V_{0}=10$, and $d_{\mathrm{p}}=1.5$. To calculate the initial conditions we solve the real Ginzburg-Landau equation (RGLE); this minimizes the energy of the fluid in the presence of the potentials modeling the particles.

\section{Magnus force model}

Lift force or the Magnus effect is a well-studied phenomenon in classical fluid dynamics [46]. In a fluid flow with a uniform upstream velocity $\mathbf{u}_{\text {flow }}$, a cylindrical disk with circulation $\Gamma$ around it experiences a lift force $\rho \Gamma \mathbf{u}_{\text {flow }} \times \hat{\mathbf{e}}_{z}$, where $\rho$ is the fluid density and $\hat{\mathbf{e}}_{z}$ is the unit vector along the cylinder corresponding to the vorticity direction. This phenomenon in superfluid was first observed by Vinen [47] by measuring modifications to frequency of a vibrating wire submerged in He II, which also allowed demonstration of the quantization of the circulation around the wire. In the present work, the fluid flow relative to a solid object is induced not by mechanical properties of the solid object itself (e.g., its elasticity), but rather by an external vortex (or multiple vortices) not trapped by this particular object.

Here, we want to explore the dynamics of an assembly of particles trapped on 2D vortices in superfluids. In particular, we want to elucidate the role of the Magnus force acting on these particles. To this end, we develop a Magnus force model (MFM) to describe this system. The Magnus force induced acceleration of the $j$ th particle trapped on a vortex (circulation strength $\Gamma)$ at the location $\left(x_{j}, y_{j}\right)$ is given by

$$
\begin{aligned}
& \ddot{x}_{j}=A\left(\dot{y}_{j}-v_{j}\right)+G_{x} /\left(m_{0}+m^{\prime}\right), \\
& \ddot{y}_{j}=A\left(u_{j}-\dot{x}_{j}\right)+G_{y} /\left(m_{0}+m^{\prime}\right),
\end{aligned}
$$

where the overhead dots indicate time derivative; $\left(u_{j}, v_{j}\right)$ is the flow velocity at the location of the $j$ th particle collectively induced by the other (excluding the $j$ th) vortices. In our description of the particle dynamics, we ignore the variation of the flow velocity over a distance comparable to the particle radius. Such an approximation is valid when the distance between the particles is large compared to both the particle radius and the healing length. The parameter

$$
A=\frac{\Gamma \rho}{\left(m_{0}+m^{\prime}\right)}
$$

is the natural oscillation frequency of the vortex trapped particle in our system. $m_{0}$ is the physical mass of the particle and $m^{\prime}$ the hydrodynamical added mass (see below). The circulation and density are a result of the Kutta-Jukowski lift forces on the particle from the fluid. The parameter $A$ corresponds to the Kelvin quasiparticle frequency of the vortex. Interestingly, the effective mass of a vortex $\left[\left(m_{0}+m^{\prime}\right)\right.$ in (8)] is nonzero even in the absence of the additional tracer particles at nonzero temperatures $[40,48]$. This is due to added mass being nonzero because the core is finite and induces drag due to the acceleration of flow around the vortex. This frequency was highlighted in the single vortex 3D case in [49].

Note that in (6) and (7) we have introduced a short-range repulsion $G$ between the particles, which acts only when the particles come to distances comparable to their size. Strictly speaking the approximation that the velocity around a particle is uniform fails at such short distances; however, our goal in this paper is to test the model beyond its formal limits of applicability in order to test its robustness. The added mass can be computed using the unsteady Bernoulli equation 
(derived from the GP equation),

$$
\frac{\partial \phi}{\partial t}+\frac{1}{2}(\nabla \phi)^{2}-\sum_{j=1}^{\mathcal{N}_{0}} V_{\mathcal{P}}\left(\mathbf{r}-\mathbf{q}_{j}\right)=\frac{1}{2} \frac{\nabla^{2} \sqrt{\rho}}{\sqrt{\rho}}-\frac{p}{\rho},
$$

where $\phi=\arg \psi$, which relates to the superfluid velocity $\mathbf{v}$ via $\mathbf{v}=\nabla \phi$, density $\rho=|\psi|^{2}$, and the first term on the right-hand side in Eq. (9) is the quantum pressure term. Note that in this model the dynamics are compressible and that $\rho=\rho(\mathbf{x}, t)$.

Let us assume for the moment that the potential representing the particle is hard, i.e., it has a well defined boundary (an extension to the case of "soft" particle potential is made later). In such a case, the superfluid density $\rho$ is zero within the particle boundary and it "heals" to its bulk value over a boundary layer that is approximately healing length wide. Now, if the particle radius $R \gg \xi$, then we can regard the particle and its boundary layer as a single moving control volume. This allows us to neglect the quantum pressure at the boundary of the considered control volume, and we can write

$$
p=-\rho\left(\frac{\partial \phi}{\partial t}+\frac{1}{2}|\nabla \phi|^{2}\right)
$$

i.e., the classical expression for irrotational ideal fluids. Thus, we can apply the classical textbook calculations for both the Magnus force and the added mass by integrating the pressure distribution over the control volume boundary [50]. If the particle under consideration is a 2D disk of radius $R$, then the $\frac{\partial \phi}{\partial t}$ term gives rise to the added mass $m^{\prime}=\rho \pi R^{2}$.

Our method of treating the dynamics of particle loaded vortices self-consistently takes into account the variations in the background flow velocity around the particles because of the dynamically varying separation between the vortices. It is important to appreciate the fact that the Magnus force description given by Eqs. (6) and (7) depends on the motion of the particle relative to the fluid and the resulting force is perpendicular to the motion. In the other words, there would be no Magnus force if the particle motion was tracing the fluid paths (this regime would be realized by the limit of very small and light particles).

In our GP simulations, we use a Gaussian potential, which has a soft boundary, to represent particles; therefore, we need an estimate of an effective radius to compute the added mass $m^{\prime}$. A consequence of the soft boundary is that the fluid can penetrate a region of the potential, but is slowed down by the increasing strength of the potential until the reflection point. A correct estimation of the effective radius is a nontrivial exercise. Therefore, we compute it in an ad hoc manner by making use of the Thomas-Fermi (TF) profile of the superfluid density around the particle potential. The TF profile is the density of a time-independent solution of Eq. (5) in which we remove the Laplacian term; this approximates the density depletion due to the particle on the surrounding fluid with no kinetic energy. The TF profile $\left|\psi_{\mathrm{TF}}\right|^{2}$ in the presence of a single particle is then given by

$$
\left|\psi_{\mathrm{TF}}\right|^{2}=\left(1-V_{0} e^{\frac{-r^{2}}{2 d_{p}^{2}}}\right) \mathcal{H}\left(r-R_{\mathrm{TF}}\right),
$$

where $\mathcal{H}$ denotes the Heaviside step function and $R_{\mathrm{TF}}$ the radius of the region within which the profile is zero. In Fig. 1 we show a slice of the TF profile, along with the initial

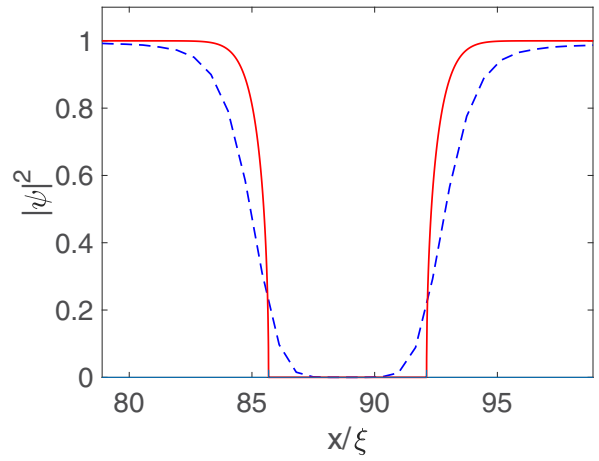

FIG. 1. Density profile of particle and TF profile: The density profile of the simulated particle is blue (dashed) and the TF profile is red, both with the same parameters, $V_{0}=10 g \rho_{0}$ and $d_{p}=1.5 \xi$.

condition. The initial condition is a slice across the particle as calculated by solving the RGLE with the potentials modeling the particles. The radius $R_{\mathrm{TF}}$, computed from the Eq. (11), is given by

$$
R_{\mathrm{TF}}=d_{p} \sqrt{2 \ln \left(V_{0}\right)} .
$$

The TF profile allows us to obtain an estimate of the displaced mass of the superfluid due to the particle (while fluid density is held fixed at $\rho_{0}$ ). We use this displaced mass as the added mass, as this captures the features of the soft potential. Thus,

$$
m^{\prime}=2 \pi \int_{0}^{\infty}\left(1-\left|\psi_{\mathrm{TF}}\right|^{2}\right) r d r .
$$

To be more clear here, the added mass can be decomposed into two parts: $m_{1}^{\prime}$, the contribution governed by the geometry of the particle, while assuming incompressibility, and $m_{2}^{\prime}$, the contribution coming from the boundary layer. We express this as follows:

$$
\begin{aligned}
m^{\prime}=m_{1}^{\prime}+m_{2}^{\prime} & =\pi R_{\mathrm{TF}}^{2}+2 \pi \int_{R_{\mathrm{TF}}}^{\infty} V_{0} e^{-\frac{r^{2}}{2 d_{p}^{2}}} r d r \\
& =\pi R_{\mathrm{TF}}^{2}+V_{0} d_{p}^{2} e^{-\frac{R_{\mathrm{TF}}^{2}}{2 d_{p}^{2}}} \\
& =d_{p}^{2}\left[2 \pi \ln \left(V_{0}\right)+1\right] .
\end{aligned}
$$

It is worth emphasising that the added mass depends on the geometry of the particle, and in the case of an arbitrarily shaped particles further considerations are required compared to what we wish to address in the present study. For the parameters used in this study, we find $m^{\prime}$ to be 46.69 .

We use the added mass $m^{\prime}$ of the displaced superfluid from the TF profile to define the ratio

$$
\mathcal{M} \equiv \frac{m_{0}}{m^{\prime}},
$$

which allows us to distinguish between heavy $(\mathcal{M}>1)$, neutral $(\mathcal{M}=1)$, and light $(\mathcal{M}<1)$ particles. We emphasise here that the added mass in general differers from the displaced mass; however, for disk shaped particles they are identical.

We express the MFM equations in a more compact form as

$$
\ddot{z}_{j}=i A\left(w_{j}-\dot{z}_{j}\right)+G /\left(m_{0}+m^{\prime}\right),
$$




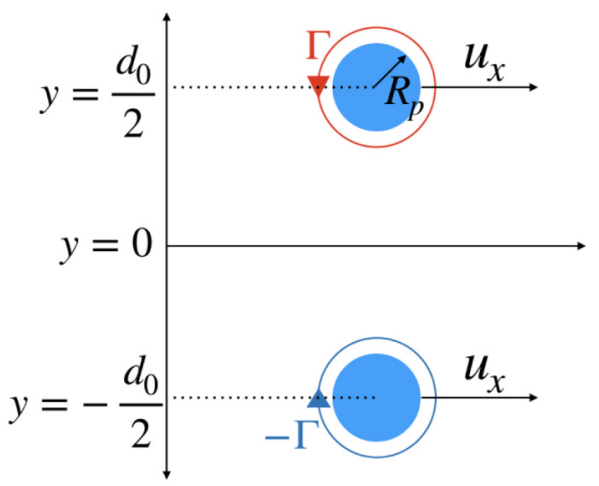

FIG. 2. Schematic diagram illustrates the initial configuration, wherein a vortex-antivortex pair loaded with particles is translating along the $x$ axis. Particles are shown by blue disks. The circle with anticlockwise (clockwise) arrow represents a vortex (an antivortex) with circulation vector pointing out of (in) the plane. The centers of the particles (the coincident vortices) are separated by a distance $d_{0}$.

where $z_{j}=x_{j}+i y_{j}, w_{j}=u_{j}+i v_{j}$ and $G=G_{x}+i G_{y}$.

We can easily extend our 2D MFM to 3D by using the local induction approximation (LIA) of the Biot-Savart law, wherein a vortex element $\mathbf{S}$ of a vortex line at the arc length $\zeta$ and time $t$ has velocity [51]

$$
\dot{\mathbf{s}}(\zeta, t)=\beta \mathbf{s}^{\prime} \times \mathbf{s}^{\prime \prime},
$$

where the overhead dot and prime correspond to derivatives with respect to the time and arclength, respectively, $\beta=$ $\ln (l / \xi)$, and $l$ is a suitable cutoff length scale that is approximately equal to the mean curvature radius. In particular this approach was used to study the modifactions of Kelvin waves in the presence of tracer particles [49].

Therefore, in 3D the dynamics of the vortex line, with uniform mass distribution, is given by

$$
\ddot{\mathbf{q}}=A \mathbf{s}^{\prime} \times[\dot{\mathbf{q}}-\dot{\mathbf{s}}(\zeta, t)],
$$

where $\mathbf{q}(\zeta, t)$ is the position of the particle. This equation corresponds to a vortex line with test particles densely filling its core. Another interpretation could be normal fluid trapped into the superfluid vortex core. Similar to the 2D case, in 3D we can include a short-range repulsion between the particles.

The LIA is derived from the more general Biot-Savart law. Using the Biot-Savart description with our model requires more complexity. This is due to the vortex arclength not being conserved in the Biot-Savart description. Thus, in general a separate equation for the mass density along the vortex line would be required.

\section{Simplified Magnus force model for particle loaded vortex-antivortex pair}

A vortex-antivortex pair of size $d_{0}$ is the simplest multivortex configuration that occurs in a periodic $2 \mathrm{D}$ domain. This vortex-antivortex pair translates at a speed $u=\Gamma /\left(2 \pi d_{0}\right)$ in a direction perpendicular to the line joining the two vortices (see Fig. 2). Therefore, the dynamics of the two particles $\mathcal{P}_{1}$ and $\mathcal{P}_{2}$ trapped on the vortex and antivortex, respectively, serves to provide a simple demonstration of the Magnus effect.
To simplify our discussion, we assume that the $y$ component of the velocity of the underlying flow experienced by the particles is zero, i.e., $v=0$ in Eqs. (6) and (7). Also, we consider a large vortex-antivortex pair, $d_{0} \gg R_{p}$, that allows us to neglect the short-range repulsion. As our vortex-antivortex pair is symmetric about the $x$ axis (See Fig. 2), in what follows we discuss the dynamics (trajectory) of only one particle. The equation of motion for the particle is then given by

$$
\begin{gathered}
\ddot{x}=-A \dot{y}, \\
\ddot{y}=-A[u(y)-\dot{x}] .
\end{gathered}
$$

To further simplify the discussion, we impose a condition that the horizontal component $u(y)=$ constant during the dynamical evolution of this system, thereby furnishing a readily solvable set of coupled ordinary differential equations (ODEs). Hereafter, we refer to this model as the simple Magnus force model (SMFM). In SMFM, with the initial conditions

$$
\begin{array}{ll}
x(0)=x_{0}, & y(0)=y_{0}, \\
\dot{x}(0)=0, & \dot{y}(0)=0,
\end{array}
$$

the particle trajectory is of the following form:

$$
\begin{aligned}
& x(t)=x_{0}+u t-\frac{u}{A} \sin (A t), \\
& y(t)=y_{0}-\frac{u}{A}[1-\cos (A t)] .
\end{aligned}
$$

Note that this rather restrictive description is valid only when the oscillations of the particles are small.

In the present study, we compare the predictions of the MFM and SMFM against the GP description to illustrate the Magnus effect. Therefore, it is important to recognize the fact that, due to the periodicity of the phase of the wave function representing the vortex-antivortex pair within the GP description in a $2 \mathrm{D}$ periodic domain, the motion of the pair is altered as compared to that in the ideal fluid. We discuss this in detail in the Appendix, where we provide a detailed comparison of the vortex-antivortex pair dynamics in the GP equation and the ideal fluid case (Weiss-McWilliams formula).

\section{RESULTS}

\section{A. Dipole configuration}

We first discuss the dynamics of the particle loaded vortexantivortex pair, starting with an initial configuration as shown in Fig. 2. We choose this configuration, rather than a single vortex and particle, to satisfy the periodic boundary conditions. It would be possible to simulate such an interaction by imposing different boundary conditions; however, we propose that the self-induced motion (i.e., velocity field) makes the dipole a more interesting example. We provide reasoning for the expected dynamics of a single vortex and particle in Sec. III B. In Fig. 3, we show the pseudocolor plots of the density field $\rho(\mathbf{r})$ overlaid with trajectories of the two particles, along with SMFM predictions (blue dashed curves) given by Eqs. (22) and (23). We find that the particles follow a nearly cycloid trajectory, in good agreement with the SMFM predictions. The cycloid trajectory of the particles is characterized by the displacement amplitude $\delta_{a}$ in the $y$ direction and 


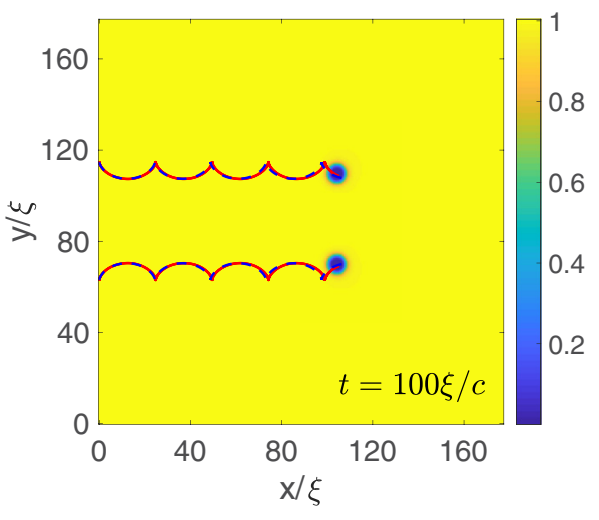

FIG. 3. Trajectories of loaded vortex dipole. Parameters: mass ratio $\mathcal{M}=17.15$ and initial separation $d_{0}=52.50 \xi$. Pseudocolor density $\left[\left|\psi\left(\mathbf{r}, t_{f}\right)\right|^{2}\right]$ plots with simulated (red) and MFM-predicted (blue dashed) trajectories overlaid.

the periodic length $X_{p}$ in the $x$-direction. These two quantities are easily deduced from the SMFM [Eqs. (22) and (23)] yielding $\delta_{a}=2 u / A$ and $X_{p} \simeq u 2 \pi / A$; note the dependence on the flow velocity $u$.

To better appreciate this dependence, we approximate the flow velocity $u$ by the following three values, with $u_{\text {pair }}$ given by Eq. (A10):

(1) $u=u_{\text {pair }}\left(d_{0}\right)$;

(2) $u=u_{\text {pair }}\left(d_{0}-2 \delta_{0}\right)$, where $\delta_{0}=2 u_{\text {pair }}\left(d_{0}\right) / A$ is the amplitude of case (1);

(3) $u$ is the mean of the estimates obtained from (1) and (2) above.

In Figs. 4(a) and 4(b) we show the plots of $\delta_{a} / d_{0}$ and $X_{p} / \xi$, respectively, vs $\mathcal{M}$ obtained from the GP equation simulations (curves with circles as markers) and the use of the above three test cases for $u$ in SMFM; we do this for two initial values of $d_{0}=52.30 \xi$ (blue curves) and $d_{0}=72.41 \xi$ (red lines). This exercise reveals a clear dependence of the results on the choice of $u$; thereby suggesting that, in any modeling scheme based on the MFM model, the flow velocity $u$ must be updated in a self-consistent manner. Note that case 1 is the velocity at the initial time; this also corresponds to the minimum velocity, as the particles are at maximum separation. Case 2, which tends to overshoot, is based on the approximate minimum distance, thus resulting in maximum velocity. Case 3 is a simple average; we see that this predicts well the parameters of the cycloid trajectories for all the simulations.

Also note that in Fig. 4 the data for $d_{0}=52.50 \xi$ and $\mathcal{M}>30.87$ are absent, as for these values of the parameters the amplitude of the cycloid motion becomes larger than half the initial vortex-antivortex pair size and results in a collision of the two particles, thereby annihilating the vortices. Later (in Fig. 7) we show that the model fits well up until the collision.

Now let us recall an assumption of our model, that the flow is uniform across the particle. This assumption is good as long as the vortex-antivortex pair size is large. However, as the size decreases, the finite size of the particles becomes increasingly important due to the external flow varying over the particle. Despite these restrictions, the analytic model provides a good phenomenological description of the dynamics of the particle loaded vortices.

To improve the simplified model we remove the assumption that the velocity is constant throughout the dynamics; namely, we let the velocity vary depending on the current separation of the particles. We solve the MFM Eqs. (6) and (7) numerically, while accounting for the variation in $u \equiv$ $u_{\text {pair }}(d(t))$ as the vortex-antivortex pair size varies; this allows us to improve the accuracy. We compare the trajectories of the particles obtained directly from the GP simulations with those predicted by the MFM. We solve the coupled ODEs of the MFM by using the specialized ODE solver ODE45 in MATLAB. We also include the short-range repulsion forces as now they are relevant for the cases where our model is tested beyond the formal limit of applicability, namely, when the inequality $\xi \ll R \ll d$ does not hold.

For comparing the above two solutions, we define an average error

$$
\epsilon=\sum_{i=1}^{N_{t}}\left\{\left[x_{s}\left(t_{i}\right)-x_{p}\left(t_{i}\right)\right]^{2}+\left[y_{s}\left(t_{i}\right)-y_{p}\left(t_{i}\right)\right]^{2}\right\}^{1 / 2} / N_{t}
$$

where $\left(x_{s}, y_{s}\right)$ are the GP simulated coordinates of the particle and $\left(x_{p}, y_{p}\right)$ are the coordinates from the simulated ODE. We choose $N_{t}$ points on each trajectory which are evenly spaced
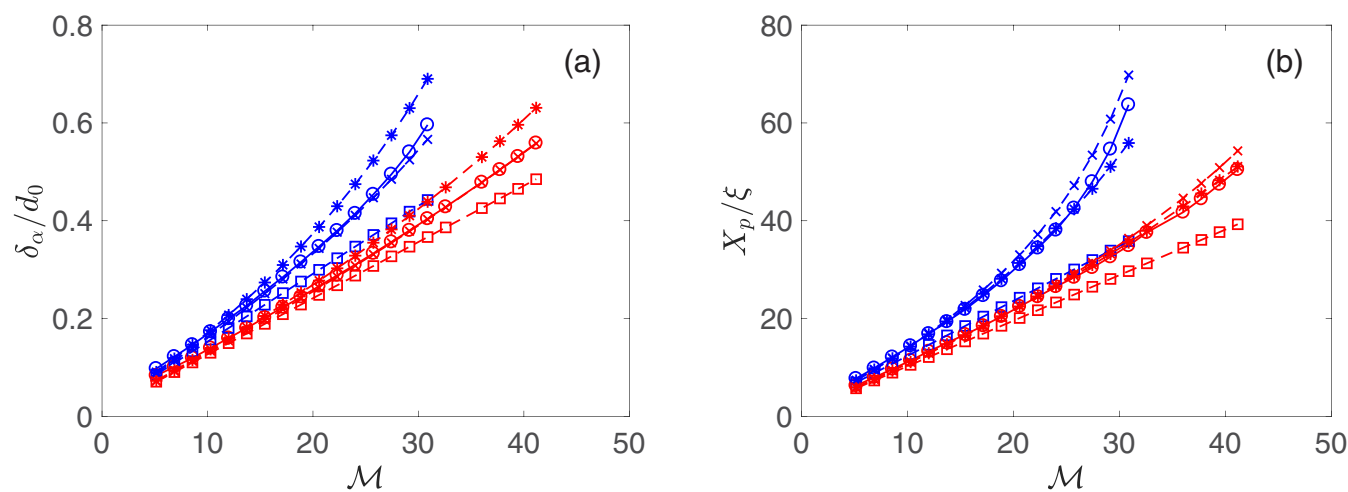

FIG. 4. Amplitude and period analysis of SMFM. All blue (top four) lines correspond to a separation $d_{0}=52.50 \xi$ and red lines (bottom four) to $d_{0}=72.41 \xi$ with the circles representing simulated data in both (a) and (b). The other types of line correspond to the fit for velocity $u_{\text {pair }}\left(d_{0}\right)$ (square, case 1 in text), for velocity $u_{\text {pair }}\left(d_{0}-2 \delta_{\alpha}\right)$ (asterisk, case 2 in text), and the average of the other two (cross, case 3 in text).

(a) The amplitude $\delta_{\alpha}$ of the cycloid in units of initial separation against mass ratio $\mathcal{M}$. (b) The period $X_{p}$ against mass ratio $\mathcal{M}$. 


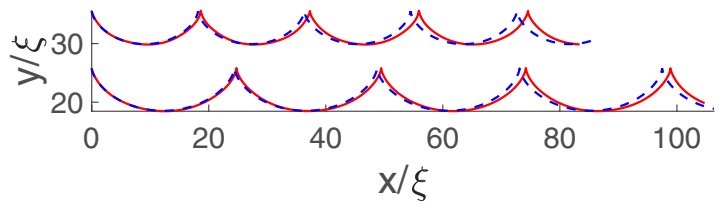

FIG. 5. Trajectories of the loaded upper vortex in the dipole. Example of DNS trajectories (red) compared to predicted trajectories (blue dashed). Two cases show $d_{0}=52.50 \xi$ (bottom curve) and $72.41 \xi$ (top curve) with $\mathcal{M}=17.15$. The final time of the simulation is $t_{f}=100$.

in time. The measure of error, $\epsilon$, is the average distance the predicted value is away from the true value over the entire run; we present this in units of $\xi$.

In Fig. 5, we present two examples $\left(d_{0} / \xi=52.50\right.$ and 72.41) of direct comparison between the particle trajectories obtained from the GP simulations and those predicted by the MFM. Note that we only show the trajectory of the particle trapped on a vortex with positive circulation because of the symmetry. The mismatch between the trajectories gives a visual indication of the error.

We perform numerical simulations for a large range of particle masses $\mathcal{M} \in[5,50]$ for the same two initial separations, keeping the total time of the dynamics fixed at $t_{f}=$ $100 \xi / c$. We then compute the error $\epsilon$ and present the results in Fig. 6(a). We observe that the error for all simulations stays of the order of a healing length. Surprisingly, we see that as the mass grows the error decreases for the pair with larger initial separation. As we increase the mass, we expect that the particle is less sensitive to compressibility effects. We also note that, for a greater mass, the amplitude of the cycloid for a given initial position is larger. We see the effect of this on the pair with smaller initial separation. The error grows rapidly as the mass approaches $\mathcal{M}=30$; at this point, the particles collide, causing the vortices to annihilate. In general, the model predicts the behavior of more massive particles more accurately as long as the inequality $\xi \ll R \ll d$ holds. However, increasing the mass causes larger amplitudes, subsequently causing the inequality to break.
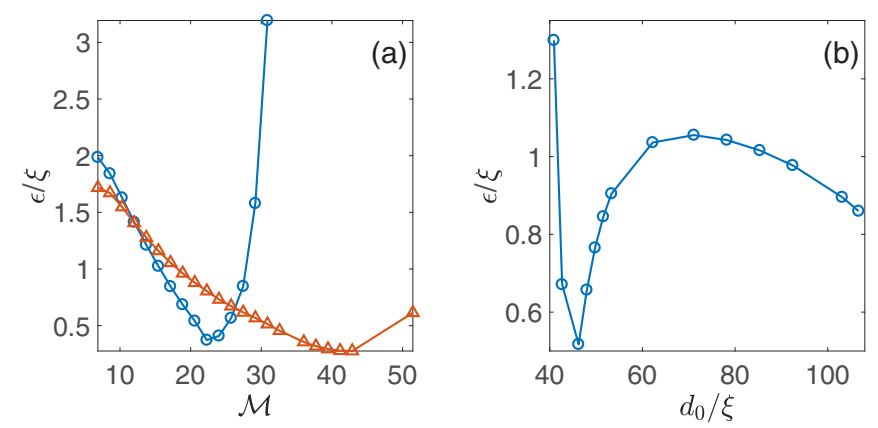

FIG. 6. Error with simple dipole configuration. (a) Two cases show $d_{0}=52.50 \xi$ (blue circles) and $72.41 \xi$ (red triangles) for a range of different masses. For values of $\mathcal{M}$ greater than 30.87 the particles have collided for $d_{0}=52.50 \xi$ (blue circles), so they have been omitted. (b) For $\mathcal{M}=17.15$ with a range of different initial distances.
In another protocol, we keep the mass fixed at $\mathcal{M}=$ 17.15 and vary the initial size of the vortex-antivortex pair. The nonlinear shape of this error is again due to competing assumptions of our model. For low separations the finite size of the particle becomes important, thus we see high error. We conjecture that the minimum that follows is due to the change in velocity due to the separation, $(\partial u / \partial d) 2 R$, becoming small compared to the velocity; see Fig. 13 in the Appendix. The error always stays of the order of a healing length. This clearly demonstrates the usefulness of a simple ODE based MFM model, which is easily solved numerically and able to capture the phenomenological motion of the vortices loaded with particles. Moreover, it predicts their motion to high accuracy.

The above examples show that the MFM model provides a good description for a simple configuration. Now we extend its use to predict more complex configurations.

As mentioned earlier, one of the limitations of our model is that it does not capture the possible annihilation of vortices during a collision event. This process plays an essential role in the dynamical evolution of an assembly of vortices in 2D in the presence of particles, which can potentially increase the annihilation rate [43]. This has implications for the quench (relaxation) dynamics of the 2D superfluid system, which undergoes the Berezinskii-Kosterlitz-Thouless (BKT) [52,53] phase transition via vortex annihilations. Consequently, the increased annihilations in 2D and vortex reconnections in $3 \mathrm{D}$ will have a strong influence the velocity statistics in a turbulent state [54].

In Fig. 7 we show that the model describes the dynamics well up until a collision in which the vortices annihilate. The annihilation is followed by linear motion of the particles, with the particles conserving their momentum from the collision. Although the current models do not account for the annihilations, this feature can be added in an ad hoc manner, for example as in Ref. [55]. To add this we require further study of the collisions to define some particle separation cutoff value for which the vortices annihilate. In order to define such a cutoff we need to study the different possible dynamics during such a collision and to further understand the exchange of momentum of the particles and the role of the acoustic component. However, this is beyond the scope of the current work.

\section{B. More complex configurations}

We studied the case of particles on vortices in a dipole configuration in detail. We now want to briefly consider further configurations to see how the model performs in more complex setups.

It is well known that vortices with more than one quanta of circulation are unstable [56,57] and move apart due to the emission of sound due to the rotational acceleration. There have been studies on the role of static potentials in stabilizing the vortices [58-61]; however, this can be extended to cases with external flows and dynamic potentials like the particles presented here. We show that the particles can be used to add stability and to create a localized region in a flow with circulation larger than one quanta.

We consider a vortex-antivortex pair configuration similar to that in Fig. 2, but where each vortex has two quanta of 

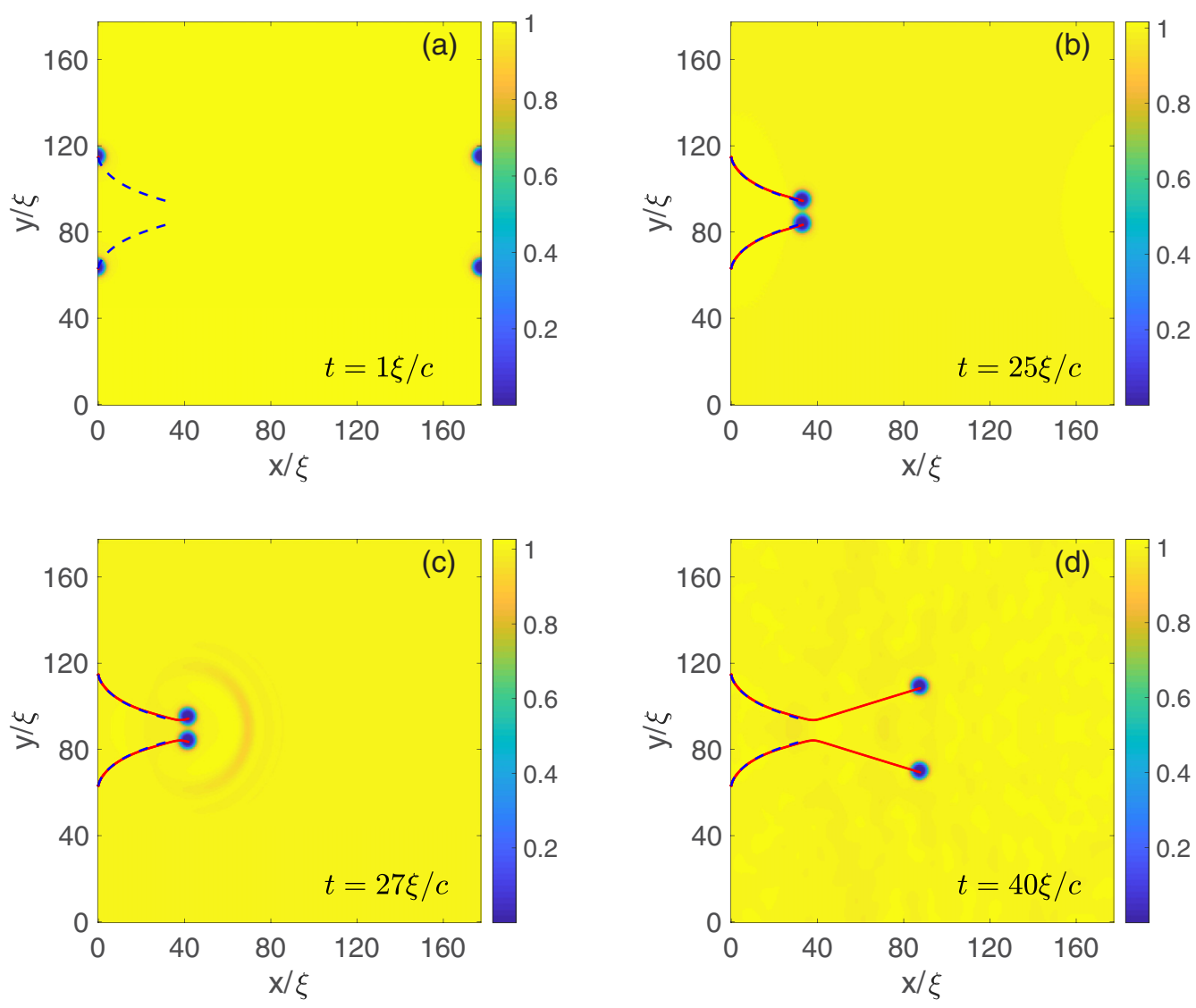

FIG. 7. Collision of loaded vortices. Parameters: mass ratio $\mathcal{M}=36.02$ and separation $d_{0}=52.5 \xi$. Pseudocolor density $\left[\left|\psi\left(\mathbf{r}, t_{f}\right)\right|^{2}\right]$ plots with DNS (red) and MFM (blue dashed) trajectories overlaid with time presented in the each panel. Sound produced during annihilation can be seen as variations in the pseudocolor density. The MFM trajectories are only presented up until the collision.

circulation and a particle trapped on it. Such a system exhibits exciting dynamics: it remains stable, i.e., both vortices remain on the particle, up until the collision. We show the collision of the particles in Fig. 7, where on colliding only one of the pairs is annihilated via the emission of an unstable RobertJones [62] soliton. The other pair remains on the particles, continuing to propagate, with the dynamics being captured by the MFM. Figure 8 shows that the MFM can successfully model the post-collision dynamics. We initialize the MFM with a position and velocity taken from the simulated GP data after the collision. Even in the presence of considerable background sound waves, we obtain an excellent agreement with the GP dynamics, with the error being $\epsilon=2.74 \xi$. The fact that the collisional interaction exhibits annihilation of only a single vortex-antivortex pair merits further investigation, as it is not trivial to understand why the entire vortical charge on the particle was not annihilated. This example also emphasizes how particles can change the dynamics of a simple configuration, and that reconnection events may be more common when vortices are loaded with particles.

We also present an example of vortex becoming trapped by a particle in Fig. 9. Here we have the same initial configuration as in Fig. 2; however, we have displaced the particle from the vortex core. The vortex is positioned such that it will collide with the particle. As the particle is trapped it quickly centers itself on the center of the vortex with any excess energy radiated as sound. The MFM ignores the self- induced velocity. However, the alignment of the vortex core and particle happens quickly due to the emission of sound. This could be added to our model algorithmically in a manner similar to what is done in Biot-Savart type models; this would include predicting the outcome of the interaction of a free vortex and a particle and applying this outcome once a particle and vortex become closer than some predefined threshold value. To apply this we would need to estimate the attractive force between the particle and the vortex. The attractive force can be understood from considering Eq. (4). Around the particle we can qualitatively separate the domain into three subdomains: 1 . Inside the TF radius, where $|\psi|^{2}=0$ so there is no contribution to the force. 2 . The region where $|\psi|^{2}=1$ and $V_{\mathcal{P}} \approx 0$; here there is not much contribution to the force on the particle. 3 . The space in between where $V_{\mathcal{P}} \approx \frac{V_{0}}{d_{p}}$ and $|\psi|^{2}$ increases with $r$. In this 3 third region we can approximate the trapping force of a vortex on a particle by assuming a vortex is a hole in the density of area $\pi \xi^{2}$. Thus the trapping force can be approximated by $f_{T} \sim \rho_{0} \pi \xi^{2} V_{0} / d_{p}$. We could then use this to modify the velocity of a particle trapped on a vortex in MFM shortly after a trapping event occurs. Although the MFM does not account for the effect of the radiated sound on the subsequent dynamics, this could also be phenomenologically added as random perturbations to the trajectories of the particles. From this reasoning we propose that in the case of a single particle and vortex the particle will always become trapped, and with any fluctuations there will 

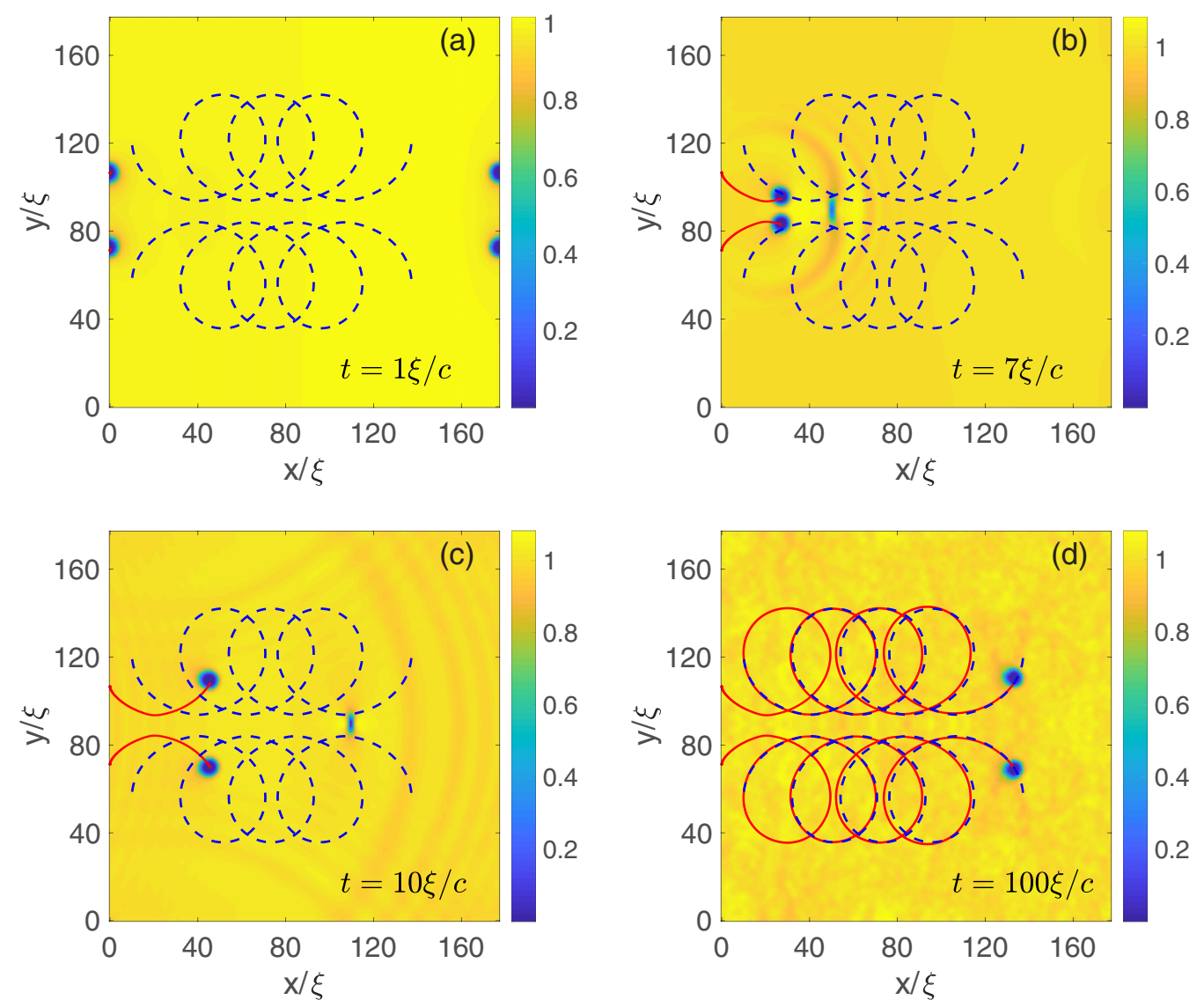

FIG. 8. Multiple vortices on particles: Pseudocolor density $\left[\left|\psi\left(\mathbf{r}, t_{f}\right)\right|^{2}\right]$ plots with DNS (red) and MFM (blue dashed) trajectories overlaid at different times as presented in the panel. The MFM trajectories are only calculated post-collision. Initially each particle has two vortices or two quanta of circulation on its core. The Robert-Jones soliton is shown by large density changes in (b) and (c).

be a restoring force realigning the center of the particle with the center of the vortex.

To further increase the complexity, we consider a final example in which free vortices (i.e., vortices not loaded with particles) are positioned in front of a vortex-antivortex pair loaded with particles; in the latter each vortex has two quanta of circulation. We compute the motion of the free vortices using (A10) such that the velocity contributions from the vortices loaded with particles are the same as if they were free vortices. The motion of the particles is once again obtained by numerically solving Eqs. (6) and (7); however, the velocity field is more complicated now. This model is more general as it can handle a combination of free vortices and vortices trapped on particles; we will refer to this as PV+MFM (point vortex and Magnus force model). We employ a simple Euler method to advance in time, with the velocity field coupling the two motions. The model predicts well the general motion not only of the particle, but also the free vortices as seen in Fig. 10. The average error of the model is $3.57 \xi$, and one can see in Fig. 10 that with the additional complexity the dynamics are captured phenomenologically.

\section{CONCLUSIONS}

We have shown that the particles trapped on vortex cores experience a Magnus force in the presence of neighboring vortices or a relative background flow. For simple configurations, where the flow velocity is known, this method can be employed to efficiently predict particle trajectories. It is also possible to generalize our model in the presence of many free vortices by accounting for their motion using the usual point vortex expressions. The MFM predictions are good enough, as long as we can ignore the finite size of the particles, e.g., for large size vortex-antivortex pairs, where it fits well even for massive particles. The model performs well outside of its formal limits of applicability.

The MFM description naturally extends to 3D by combining the model with the LIA; we hope to further develop the method beyond LIA and incorporate the full BS description in future work. Our model can be generalized to include cases in which annihilation takes place and, of course, describes well the post-collision dynamics. Further work could focus on how the attractive forces added to vortices can increase vortex annihilations and further aid phase transitions like the BKT phase transition; similar ideas have been discussed in [54]. The development of a simple theoretical framework which accounts for the motion of particles in the presence of vortices would significantly improve the ability to study the effect of particles in superfluid turbulence. We could algorithmically add annihilations of vortices and vortices on particles using similar, commonly used methods [63]; this will be the subject of future work. To do this, we would need to choose some physical cutoff value of the particle separation for which vortices annihilate. This could be found by further studies 

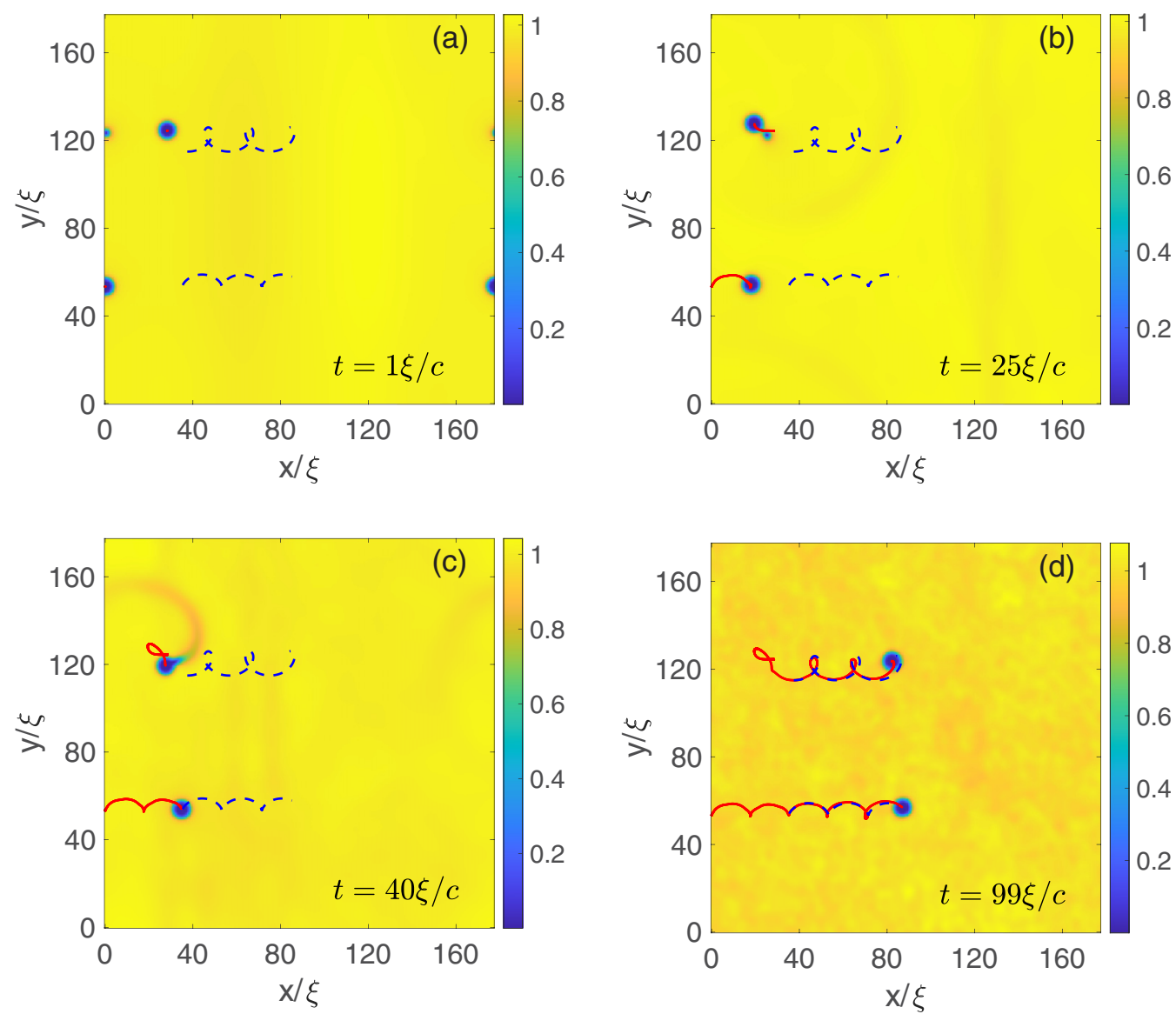

FIG. 9. Free vortices being trapped by a particle. Parameters: mass ratio is $\mathcal{M}=17.15$ and initial separation $d_{0}=52.5 \xi$. Pseudocolor density $\left[\left|\psi\left(\mathbf{r}, t_{f}\right)\right|^{2}\right]$ plots with DNS (red) and MFM (blue dashed) trajectories of particles and free vortices overlaid. The smaller disks in the pseudocolor density plots are free vortices. The DNS (red) and MFM (blue) are overlaid and are difficult to distinguish.

into the annihilation of vortices in the presence of external potentials. However, with the current model we have a fast and accurate way to simulate $2 \mathrm{D}$ turbulence interacting with tracer particles.

The physics that emerges from such a treatment of active particles could also be qualitatively relevant for the studies of stabilization of a vortex in a harmonically trapped BEC. For example, noncondensate atoms could become trapped on the vortex core, leading to the stabilization of the vortex at finite temperatures $[64,65]$. Similarly, the dynamics of a vortex which has been stabilized by providing a pinning potential via a focused laser beam at the vortex core could be modeled by our approach; however, in such a case the resulting vortex dynamics would have a prescribed component and not be completely active. Furthermore, if we consider two different species of BECs we would expect to see a similar phenomenon. The dynamics in such a situation may become interesting as not only vortices will accrue particles of a second species, but also other compressible structures such as solitons will accrue particles.

\section{ACKNOWLEDGMENTS}

S.N. is supported by the Chaire D'Excellence IDEX (Initiative of Excellence) awarded by Université de la Côte d'Azur, France. M.-E.B. was supported by Agence Nationale de la Recherche through the project QUTE-HPC ANR-18CE46-0013. This project has received funding from the European Union's Horizon 2020 Research and Innovation Programme under Grant Agreements No. 823937 in the framework of the Marie Skodowska-Curie HALT project and No. 820392 in the FET Flagships PhoQuS project. Computations were carried out on the High Performance Computing Cluster and supported by the Scientific Computing Research Technology Platform at the University of Warwick. We also acknowledge the following computing grant: GENCI (Grand Equipment National de Calcul Intensif) No. A0062A10441. We acknowledge the useful discussion and contribution to this work by the reviewers.

\section{APPENDIX: WEISS-MCWILLIAMS FORMULAS FOR IDEAL FLUIDS AND GP SYSTEM}

The adaptation of the Weiss-McWilliams formula for the translational velocity of a vortex-antivortex pair in an ideal fluid to the GP system with a periodic domain requires a modification because of the periodicity of the phase of the wave function in the latter. Below we demonstrate this for a vortex-antivortex pair, which we then generalize to the case of $N$ pairs. A similar adaption has been made in [66] based on Ramanujan's formula. Here, we present a simpler, more intuitive argument and highlight the case of a vortex dipole. 

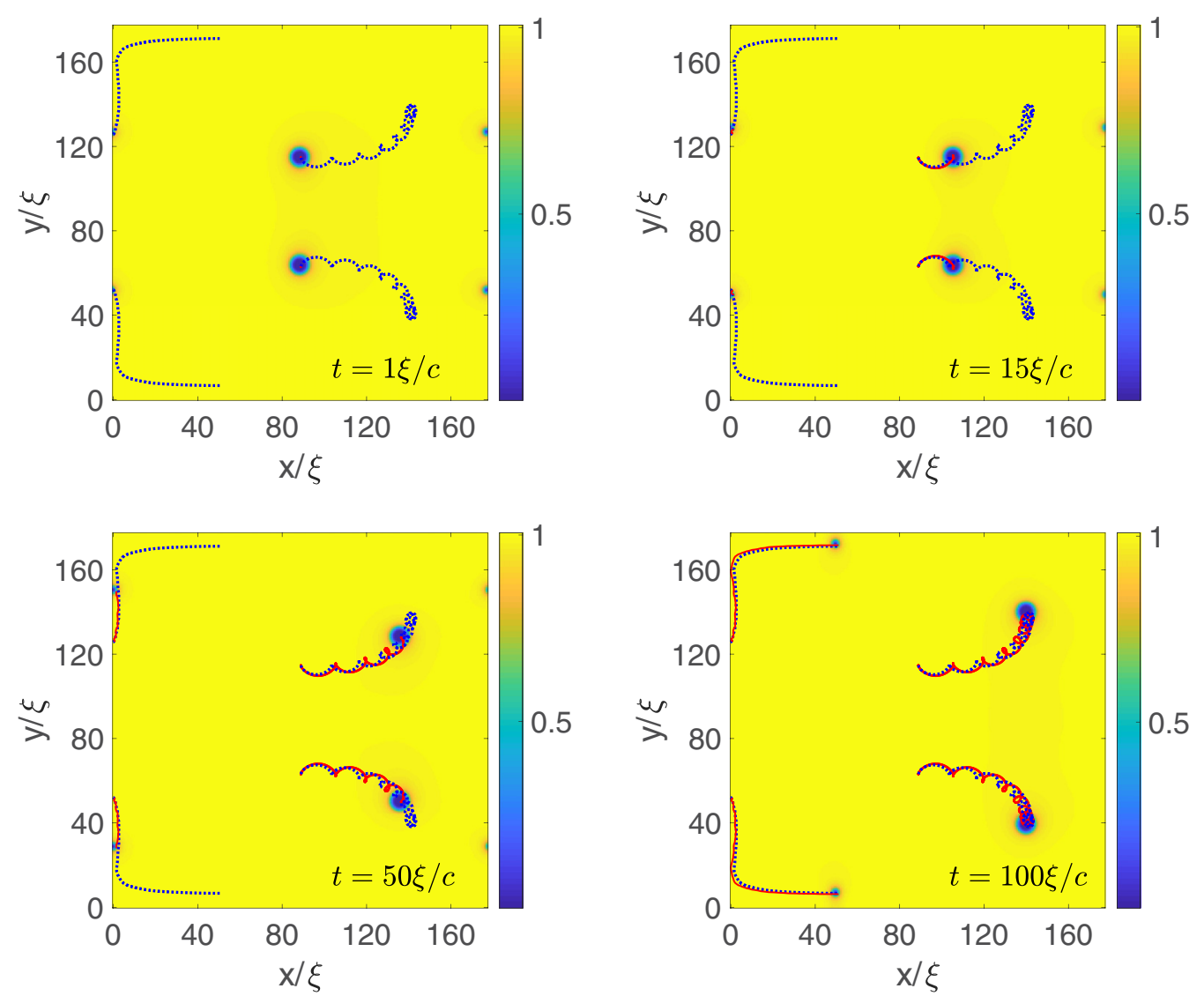

FIG. 10. Free vortices and loaded vortices. Parameters: mass ratio is $\mathcal{M}=17.15$ and initial separation $d_{0}=52.5 \xi$. Pseudocolor density $\left[\left|\psi\left(\mathbf{r}, t_{f}\right)\right|^{2}\right]$ plots with DNS (red) and PV+MFM (blue dotted) trajectories of particles and free vortices overlaid. The smaller disks in the pseudocolor density plots are free vortices. The DNS (red) and PV+MFM (blue dotted) are overlaid and are difficult to distinguish.

We also show below that the velocity shift is not necessarily small compared to the dipole velocity.

Consider a vortex-antivortex pair, translating along the $x$ axis with a vortex at $(x, \pi+d / 2)$ and an antivortex at $(x, \pi-$ $d / 2$ ). Now let us consider the circulation along three lines $\mathcal{C}_{i}$ for $i=1,2,3$, as shown in Fig. 11:

$$
\mathcal{C}_{i}(d)=\int_{0}^{2 \pi} u\left(x, y_{i}\right) d x
$$

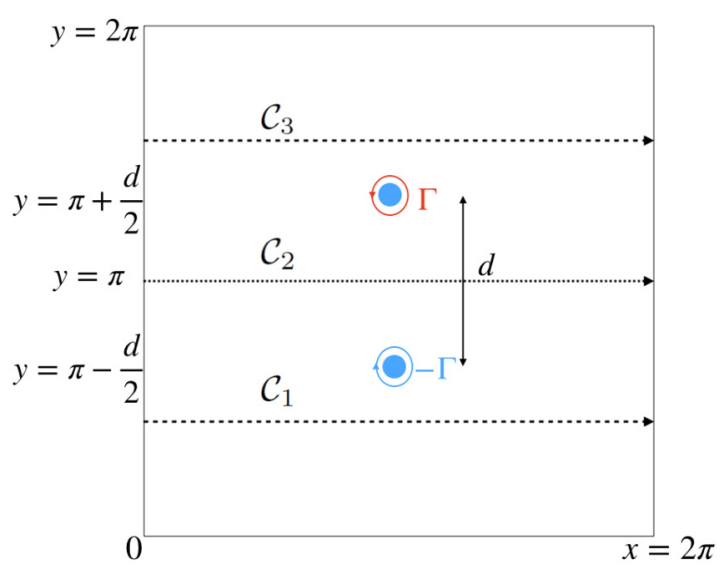

FIG. 11. Schematic of two vortices separated by distance $d$, overlaid with the contours on which we calculate the $x$ contributions of the circulation. where $0<d<2 \pi, \quad 0 \leqslant y_{1}<\pi-d / 2, \quad \pi-d / 2<y_{2}<$ $\pi+d / 2$, and $\pi+d / 2<y_{3} \leqslant 2 \pi$. These contours can be closed by joining $x=0$ and $x=2 \pi$, without generating any contribution to the circulation, as guaranteed by the periodic boundary condition. Thence,

$$
\begin{gathered}
\mathcal{C}_{1}-\mathcal{C}_{2}=-\Gamma, \\
\mathcal{C}_{2}-\mathcal{C}_{3}=\Gamma, \\
\mathcal{C}_{1}=\mathcal{C}_{2}-\Gamma=\mathcal{C}_{3},
\end{gathered}
$$

for the contours enclosing vortices. The circulation can now be solely expressed as a function of the $y$ position of the contour and the separation between the vortices:

$$
\int_{0}^{2 \pi} u(x, y) d x=\mathcal{C}(y, d)=\int_{0}^{2 \pi}-\frac{\partial h}{\partial y} d x,
$$

where $h$ is the stream function and $u=-\partial h / \partial y$. Let us define the mean velocity

$$
\begin{aligned}
\bar{u} & =\frac{1}{(2 \pi)^{2}} \int_{0}^{2 \pi} \mathcal{C}(y, d) d y \\
& =\frac{1}{(2 \pi)^{2}} \int_{0}^{2 \pi} \int_{0}^{2 \pi} u(x, y) d x d y .
\end{aligned}
$$

From the periodicity of stream function it follows that the mean velocity is zero. The integral in (A7) can be then 


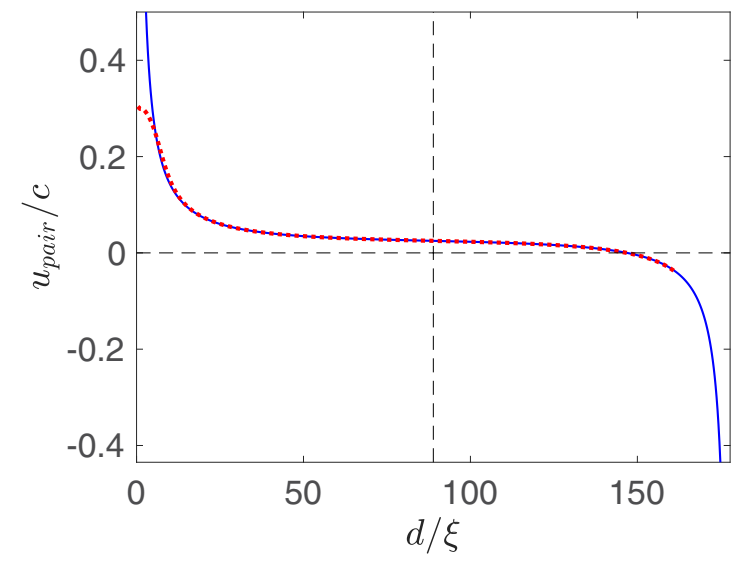

FIG. 12. Velocity of the vortex-antivortex pair as a function of its size. The red dashed curve represents the data obtained from the GP simulations, whereas the blue solid line indicates the velocity given by the adapted Weiss-McWilliams formula; see text for more details. The black dashed horizontal line indicates $u_{\text {pair }}=0$ and the black dashed vertical line marks the midpoint of the periodic domain.

computed as a sum of the areas multiplied by the circulation, that is,

$$
\begin{aligned}
\left(\pi-\frac{d}{2}\right) \mathcal{C}_{1}+\mathcal{C}_{2} d+\left(\pi-\frac{d}{2}\right) \mathcal{C}_{3} & =2 \pi \mathcal{C}_{1}+\Gamma d \\
& =0
\end{aligned}
$$

by using Eq. (A4). Thus $\mathcal{C}_{1}=-\Gamma d /(2 \pi)$ for a system with a periodic stream function. Note that the Euler equations are Galilean invariant and any constant velocity can be added to the system, which corresponds to moving to a different inertial frame. However, the requirement that the stream function is periodic fixes the frame of reference such that the mean velocity is zero. This is the choice of frame in Ref. [67]. Such a choice is inconsistent with the periodicity of the wave function, as it would result in a phase change which is not an integer multiple of $2 \pi$ (for $\Gamma=2 \pi, \mathcal{C}_{1}=d \neq 2 \pi n$ ). Therefore, if we want to use the Weiss-McWilliams prescribed velocity (ideal fluid case), we must work in a frame in which the phase of the wave function is periodic. Thus, we must add a constant background velocity equal to $\mathcal{C}_{1} / 2 \pi$.

Note that in order to be consistent with the contents of the main text, in our discussion here we have used a vortexantivortex pair that is only separated along the $y$ direction. However, our arguments are still valid if the vortices are separated in the $x$ direction as well. In that case, we simply have to repeat the argument with vertical contours which separate the vortices to find the contribution to the $y$ component of the velocity.

Moreover, our discussion here is generalizable to the case of $2 N$ vortices, with an equal number of vortices and antivortices. This is so because the system is linear, i.e., the contribution to the velocity field is additive. Also, the argument is valid, as the sum of the periodic phases will also be periodic. Therefore, for a system of $2 N$ vortices, the additional

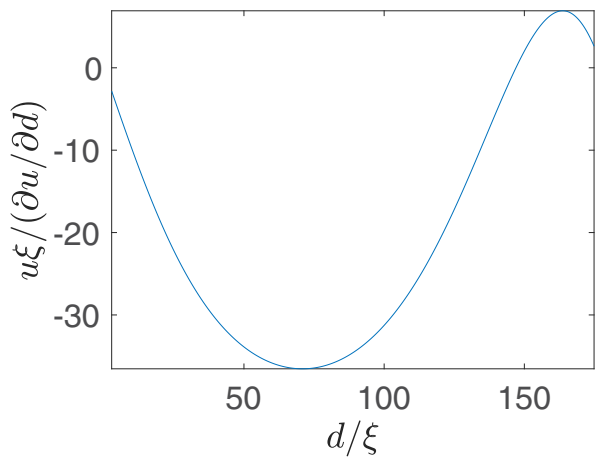

FIG. 13. Derivative of velocity with respect to separation against separation.

background velocity $\mathbf{u}_{\mathbf{b}}$ is

$$
\mathbf{u}_{\mathbf{b}}=\sum_{i=1}^{2 N} \frac{\Gamma_{i}}{(2 \pi)^{2}} \hat{\mathbf{e}}_{z} \times \mathbf{x}_{i}
$$

where $\mathbf{x}_{i}$ is the position of the $i$ th vortex, which is, up to a constant, the total momentum of the point vortex system. We note here that the velocity shift is not necessarily small compared to the average vortex velocity. In systems of randomly positioned vortices one would expect the shift to be small compared to the mean velocity $\sim \Gamma / 2 \pi d_{i v}$, where $d_{i v}$ is the mean intervortex distance [due to the cancellations in (A9)]. However, in a system that is polarized such that the positive and negative vortices form large clusters, the shift maybe be larger than the velocity of the clusters depending on the separation $\left(\left|\mathbf{u}_{\mathbf{b}}\right| \sim \frac{\Gamma N d}{4 \pi^{2}}>v_{\text {cluster }} \sim \frac{N \Gamma[1+\cos (d)]}{4 \pi \sin (d)}\right.$ for $2 \lesssim d \lesssim 5.2$ ).

For an odd number of vortices, the system will have a net circulation. Therefore, to make arguments, we now have to work in a rotating frame of reference. This is the same as adding a constant background vorticity, such that the frame has net circulation zero. The ratio between the angular velocity of the rotating frame and the vorticity is $1 / 2$. Other valid configurations could include constant vorticity such as shear. In this case, such a formulation is consistent when periodicity is imposed in sheared coordinates.

In Fig. 12 we show the comparison of the vortex-antivortex pair velocity as obtained from the GP equation DNS (dashed red curve) and the adapted Weiss-McWilliams formula (blue curve). For very small pair size, we observe a disagreement, as now the vortex core size is important. At still very small sizes, vortices annihilate and become a localized density perturbations. The original Weiss-Mcwilliams (ideal fluid) $x$-directional velocity of a vortex pair (as in 11) is given by

$$
u_{W M c}(d)=-\frac{\Gamma}{4 \pi} \sum_{n=-\infty}^{\infty} \frac{\sin (d)}{\cosh (2 \pi n)-\cos (d)} .
$$

Therefore, the expression obtained by adapting this to the case of the GP equation in a periodic domain is given by

$$
u_{\mathrm{pair}}(d)=-\frac{\Gamma}{4 \pi}\left(\sum_{n=-\infty}^{\infty} \frac{\sin (d)}{\cosh (2 \pi n)-\cos (d)}+\frac{d}{\pi}\right) .
$$


For our purposes, we calculate the velocity until it has converged within $10^{-9}$, this corresponds to retaining nine terms, i.e., $n=-4$ to $n=4$. Therefore, to the leading order,

$$
u_{\mathrm{pair}}(d)=\frac{\Gamma}{4 \pi}\left(\frac{1+\cos (d)}{\sin (d)}+\frac{d}{\pi}\right)
$$

[1] J. Maurer and P. Tabeling, Europhys. Lett. 43, 29 (1998).

[2] S. N. Fisher, A. J. Hale, A. M. Guénault, and G. R. Pickett, Phys. Rev. Lett. 86, 244 (2001).

[3] E. A. L. Henn, J. A. Seman, G. Roati, K. M. F. Magalhães, and V. S. Bagnato, Phys. Rev. Lett. 103, 045301 (2009).

[4] T. Byrnes, N. Y. Kim, and Y. Yamamoto, Nat. Phys. 10, 803 (2014).

[5] G. Baym, R. I. Epstein, and B. Link, Physica B 178, 1 (1992).

[6] L. Warszawski, A. Melatos, and N. G. Berloff, Phys. Rev. B 85, 104503 (2012).

[7] C. Michel, O. Boughdad, M. Albert, P.-É. Larré, and M. Bellec, Nat. Commun. 9, 2108 (2018).

[8] R. J. Donnelly, Quantized Vortices in Helium II (Cambridge University Press, Cambridge, 1991).

[9] A. C. White, B. P. Anderson, and V. S. Bagnato, Proc. Natl. Acad. Sci. USA 111, 4719 (2014).

[10] M. Tsubota, T. Araki, and C. F. Barenghi, Phys. Rev. Lett. 90, 205301 (2003).

[11] R. P. Feynman, in Progress in Low Temperature Physics, edited by C. J. Gorter (Elsevier, Amsterdam, 1955), Vol. 1, pp. 17-53.

[12] C. Nore, M. Abid, and M. M. Brachet, Phys. Rev. Lett. 78, 3896 (1997).

[13] C. Nore, M. Abid, and M. E. Brachet, Phys. Fluids 9, 2644 (1997).

[14] G. P. Bewley, M. S. Paoletti, K. R. Sreenivasan, and D. P. Lathrop, Proc. Natl. Acad. Sci. USA 105, 13707 (2008).

[15] K. W. Schwarz, Phys. Rev. B 31, 5782 (1985).

[16] G. Krstulovic, Phys. Rev. E 86, 055301(R) (2012).

[17] A. W. Baggaley and J. Laurie, Phys. Rev. B 89, 014504 (2014).

[18] E. Fonda, D. P. Meichle, N. T. Ouellette, S. Hormoz, and D. P. Lathrop, Proc. Natl. Acad. Sci. USA 111, 4707 (2014).

[19] V. Shukla, P. D. Mininni, G. Krstulovic, P. C. di Leoni, and M. E. Brachet, Phys. Rev. A 99, 043605 (2019).

[20] L. Skrbek and W. Vinen, in Progress in Low Temperature Physics (Elsevier, Amsterdam, 2009), Vol. 16, pp. 195-246.

[21] S. Nazarenko and M. Onorato, Physica D 219, 1 (2006).

[22] R. Desbuquois, L. Chomaz, T. Yefsah, J. Léonard, J. Beugnon, C. Weitenberg, and J. Dalibard, Nat. Phys. 8, 645 (2012).

[23] T. W. Neely, A. S. Bradley, E. C. Samson, S. J. Rooney, E. M. Wright, K. J. H. Law, R. Carretero-González, P. G. Kevrekidis, M. J. Davis, and B. P. Anderson, Phys. Rev. Lett. 111, 235301 (2013).

[24] S. P. Johnstone, A. J. Groszek, P. T. Starkey, C. J. Billington, T. P. Simula, and K. Helmerson, Science 364, 1267 (2019).

[25] G. Gauthier, M. T. Reeves, X. Yu, A. S. Bradley, M. A. Baker, T. A. Bell, H. Rubinsztein-Dunlop, M. J. Davis, and T. W. Neely, Science 364, 1264 (2019).

[26] A. S. Bradley and B. P. Anderson, Phys. Rev. X 2, 041001 (2012). and its derivative with respect to separation (Fig. 13) is

$$
\frac{\partial u_{\text {pair }}(d)}{\partial d}=-\frac{\Gamma}{4 \pi}\left(\frac{1+\cos (d)}{\sin ^{2}(d)}+\frac{1}{\pi}\right) .
$$

This has a minimum at $d=\pi$, thus the approximation of constant velocity across the particle is most suitable close to half the domain size, $L / 2$.
[27] V. Shukla, M. Brachet, and R. Pandit, New J. Phys. 15, 113025 (2013).

[28] V. Shukla, A. Gupta, and R. Pandit, Phys. Rev. B 92, 104510 (2015).

[29] C. F. Barenghi, V. S. L'vov, and P.-E. Roche, Proc. Natl. Acad. Sci. USA 111, 4683 (2014).

[30] W. Guo, M. La Mantia, D. P. Lathrop, and S. W. Van Sciver, Proc. Natl. Acad. Sci. USA 111, 4653 (2014).

[31] R. J. Donnelly and P. H. Roberts, Proc. R. Soc. London A 312, 519 (1969).

[32] G. A. Williams, K. DeConde, and R. E. Packard, Phys. Rev. Lett. 34, 924 (1975).

[33] G. P. Bewley, D. P. Lathrop, and K. R. Sreenivasan, Nature (London) 441, 588 (2006)

[34] D. Duda, P. Švančara, M. La Mantia, M. Rotter, and L. Skrbek, Phys. Rev. B 92, 064519 (2015).

[35] D. E. Zmeev, F. Pakpour, P. M. Walmsley, A. I. Golov, W. Guo, D. N. McKinsey, G. G. Ihas, P. V. E. McClintock, S. N. Fisher, and W. F. Vinen, Phys. Rev. Lett. 110, 175303 (2013).

[36] N. G. Berloff and P. H. Roberts, Phys. Rev. B 63, 024510 (2000).

[37] E. Varga, C. F. Barenghi, Y. A. Sergeev, and L. Skrbek, J. Low Temp. Phys. 183, 215 (2016).

[38] N. B. Jørgensen, L. Wacker, K. T. Skalmstang, M. M. Parish, J. Levinsen, R. S. Christensen, G. M. Bruun, and J. J. Arlt, Phys. Rev. Lett. 117, 055302 (2016).

[39] E. P. Gross, Nuovo Cimento 20, 454 (1961).

[40] L. P. Pitaevskii, Sov. Phys. JETP 13, 451 (1961).

[41] V. Shukla, M. Brachet, and R. Pandit, Phys. Rev. A 94, 041602(R) (2016).

[42] R. Pandit, D. Banerjee, A. Bhatnagar, M. Brachet, A. Gupta, D. Mitra, N. Pal, P. Perlekar, S. S. Ray, V. Shukla et al., Phys. Fluids 29, 111112 (2017).

[43] V. Shukla, R. Pandit, and M. E. Brachet, Phys. Rev. A 97, 013627 (2018).

[44] D. Acheson and F. Acheson, Elementary Fluid Dynamics (Clarendon, Oxford, 1990).

[45] http://www.fftw.org.

[46] D. Tritton, Physical Fluid Dynamics (Clarendon, Oxford, 1988).

[47] W. F. Vinen, Proc. R. Soc. London A 260, 218 (1961).

[48] T. Simula, Phys. Rev. A 97, 023609 (2018).

[49] U. Giuriato, G. Krstulovic, and S. Nazarenko, arXiv:1907.01111.

[50] H. Lamb, Hydrodynamics (Cambridge University Press, Cambridge, 1916).

[51] K. W. Schwarz, Phys. Rev. B 38, 2398 (1988).

[52] V. Berezinskii, Sov. Phys. JETP 32, 493 (1971).

[53] J. M. Kosterlitz and D. J. Thouless, J. Phys. C 6, 1181 (1973) 
[54] U. Giuriato, G. Krstulovic, and D. Proment, J. Phys. A: Math. Theor. 52, 305501 (2019).

[55] A. Baggaley and C. Barenghi, Phys. Rev. B 83, 134509 (2011).

[56] Y. Shin, M. Saba, M. Vengalattore, T. A. Pasquini, C. Sanner, A. E. Leanhardt, M. Prentiss, D. E. Pritchard, and W. Ketterle, Phys. Rev. Lett. 93, 160406 (2004).

[57] M. Möttönen, T. Mizushima, T. Isoshima, M. M. Salomaa, and K. Machida, Phys. Rev. A 68, 023611 (2003).

[58] R. P. Teles, F. A. dos Santos, and V. S. Bagnato, arXiv:1505.06227 (2015).

[59] S. Gustafson and F. Ting, J. Nonlinear Sci. 19, 341 (2009).

[60] T. P. Simula, S. M. M. Virtanen, and M. M. Salomaa, Phys. Rev. A 65, 033614 (2002).
[61] D. J. Dean and T. Papenbrock, Phys. Rev. A 65, 043603 (2002).

[62] C. A. Jones and P. H. Roberts, J. Phys. A: Math. General 15, 2599 (1982).

[63] A. W. Baggaley and C. F. Barenghi, J. Low Temp. Phys. 166, 3 (2012).

[64] R. J. Dodd, K. Burnett, M. Edwards, and C. W. Clark, Phys. Rev. A 56, 587 (1997).

[65] T. Isoshima and K. Machida, Phys. Rev. A 59, 2203 (1999).

[66] T. P. Billam, M. T. Reeves, B. P. Anderson, and A. S. Bradley, Phys. Rev. Lett. 112, 145301 (2014).

[67] J. B. Weiss and J. C. McWilliams, Phys. Fluids A 3, 835 (1991). 